\title{
Cambodge : la guerre, toujours la guerre!
}

\author{
François Guilbert
}

\section{Q OpenEdition}

\section{Journals}

Édition électronique

URL : http://journals.openedition.org/conflits/74

DOI : $10.4000 /$ conflits.74

ISSN : $1777-5345$

\section{Éditeur :}

CCLS - Centre d'études sur les conflits lilberté et sécurité, L'Harmattan

\section{Édition imprimée}

Date de publication : 21 janvier 1990

ISSN : 1157-996X

\section{Référence électronique}

François Guilbert, «Cambodge : la guerre, toujours la guerre! », Cultures \& Conflits [En ligne], 01 | hiver 1990, mis en ligne le 30 décembre 2002, consulté le 30 mars 2021. URL : http:// journals.openedition.org/conflits/74 ; DOI : https://doi.org/10.4000/conflits.74

Ce document a été généré automatiquement le 30 mars 2021.

Creative Commons License 


\title{
Cambodge : la guerre, toujours la guerre!
}

\author{
François Guilbert
}

Le danger khmer rouge

1 Depuis l'échec de la Conférence de Paris durant l'été 1989 et après le "retrait officiel" de l'armée vietnamienne (26 septembre 1989), les opérations de guerre, à l'intérieur du Cambodge, se sont poursuivies et même multipliées. Pour la première fois depuis l'établissement du protectorat français en 1863, les Cambodgiens s'affrontent entre eux sans que cela soit un enjeu stratégique planétaire ni même seulement régional. $\mathrm{Au}$ demeurant, la guerre continue sans qu'aucun des protagonistes ne puisse remporter la victoire décisive. Toutefois, les rares étrangers de passage au Cambodge disent, à la quasi-unanimité, qu'il est bien difficile de s'imaginer que le pays est en guerre. En effet, seul, le couvre-feu de $21 \mathrm{~h}$ à $5 \mathrm{~h}$ du matin et les réticences des responsables de l'État du Cambodge à délivrer aux hôtes étrangers des autorisations à se rendre à Battambang, Kampot ou Koh Kong montrent que la situation dans ces provinces est loin d'être sans danger. Preuve s'il en fallait de cette atmosphère belliciste, la radio khmère rouge a repris, depuis la mi-octobre, après la trêve consécutive à l'annonce d'un accord entre les factions cambodgiennes début septembre 1990 à Djakarta, sa diffusion de communiqués militaires. Pourtant, lors de la signature par les cinq membres permanents du Conseil de Sécurité de l'ONU d'un accord de paix le 28 août 1990, beaucoup espéraient que l'on verrait rapidement la fin des combats. Aujourd'hui, c'est l'inverse qui se passe et chaque composante de la résistance a les moyens de poursuivre ses offensives, au moins pour la prochaine saison sèche 1990-1991. A eux seuls, les Khmers rouges disposent de caches d'armes pouvant leur permettre de continuer les combats, de manière autonome, pendant deux ou trois ans. Si les discussions d'Harbin (Chine) entre les ministres des Affaires étrangères chinois et soviétique ont réellement abouti, à la fin décembre 1990, les livraisons aux deux principaux fournisseurs des belligérants devraient être suspendues. Mais, des forces en présence, c'est le gouvernement, de Phnom Penh qui a le plus à perdre dans ces marchandages. 
2 Dans le même temps, ces deux dernières années, les capacités militaires des Khmers rouges se sont considérablement renforcées. Sachant que la période de saison sèche est propice à l'utilisation de matériel lourd, chacun attend de voir si, cette année, les 24 chars légers chinois, prétendument livrés aux Khmers rouges entreront en action. A Phnom Penh, pour le moment, on fait semblant de ne pas y croire. Disposant d'une puissance aérienne limitée, pour la première fois de son histoire, l'administration de Phnom Penh pourrait bien se retrouver sur le champ de bataille avec une puissance de feu moins importante que ses adversaires. De plus, grâce aux matériels généreusement fournis par Beijing et à la bienveillante neutralité des autorités de Bangkok, les Khmers rouges bénéficient aussi d'importantes complicités occidentales qui leur permettent de renforcer leur dispositif. Si l'on en croit un journaliste d'outre-Manche ${ }^{1}$, une unité secrète d'ex-SAS ${ }^{2}$, dont les membres continueraient à rendre compte de leurs actions au QG du régiment à Hereford, formeraient en Thaïlande et en Malaysia des hommes de Pol Pot aux techniques de (dé)minage et de terrorisme (plastiquage de ponts). Profitant de ces nouvelles dispositions matérielles et du retrait de l'essentiel du contingent vietnamien, depuis novembre 1989, les soldats du GCKD ont réussi à se réinstaller dans la zone montagneuse des Phnom Malaï d'où ils avaient été expulsés par les offensives khmèro-vietnamiennes de 1984-1985. C'est d'ailleurs dans cette région du pays que, le 16 septembre dernier, plus de 50 chefs militaires Khmers rouges se sont rencontrés pour décider de la stratégie à suivre ${ }^{3}$. Face à cela, et voulant reconquérir le terrain perdu, pour ne pas affaiblir les positions de Phnom Penh dans les discussions intercambodgiennes, l'armée régulière a provoqué, dans les campagnes, un nouvel exode vers la frontière thaïlandaise. Au cours du premier semestre 1990, on estime que 40000 personnes se sont ainsi réfugiées dans les camps de la résistance khmère rouge et non communiste. De facto, toutes ces "personnes déplacées" sont prises en otage par les factions de la résistance car ce sont, aussi bien des réservoirs importants de combattants que la principale source de légitimité des mouvements armés. C'est pourquoi toutes les factions ont accueilli fraîchement la proposition du Premier ministre thaïlandais visant à créer un camp neutre à l'intérieur de son pays. Certes, l'opération risque de coûter fort cher - 4 milliards de francs -, mais l'enjeu politique du contrôle des camps est d'un prix beaucoup plus considérable. En attendant, l'aide internationale risque de servir aux opérations militaires, avant de bénéficier au rapatriement des réfugiés. Ainsi l'US Aid vient de permettre la construction, sur une vingtaine de kilomètres, d'une route stratégique reliant le camp de Site 2 à la "zone libérée" de Thmar Puok (FNLPK)4 . Quant aux Chinois, ils ne cachent pas leur souhait de voir les camps de réfugiés rester en l'Etat, au moins jusqu'aux élections. Ils sont d'autant plus déterminé à maintenir le statu quo qu'il est fort peu probable que l'administration de Phnom Penh puisse être démantelée dans son ensemble d'ici là. Toutefois, en l'absence d'un véritable camp neutre, il est à craindre que les populations civiles ne soient contraintes à retourner à l'intérieur du pays et à suivre les factions de la résistance vers leurs "zones libérées". Toutes les factions de la résistance pratiquent ces mouvements forcés de population gour montrer aux grandes puissances engagées dans la recherche d'une solution négociée au conflit qu'ils ont une véritable légitimité et qu'ils contrôlent une partie du territoire. C'est pourquoi, depuis quelques mois, les formations de la résistance, y compris les Khmers rouges, ont tenté d'approcher les organisations humanitaires présentes dans les camps à la frontière pour envisager avec elles dans quelle mesure elles seraient prêtes à gérer des programmes d'assistance aux populations civiles dans les zones libérées. En 1990, entre 60000 et 100000 personnes 
ont ainsi été "réinstallées" à I'intérieur du Cambodge, dans des conditions souvent sommaires et sans avoir eu leur mot à dire. A titre d'exemple, près du tiers des réfugiés du camp khmer rouge du Site $K$ ont déjà traversé la frontière tandis que les responsables du camp d'O Trao affirment que 20.000 personnes de leur camp seront "rapatriées" avant la fin de l'année 1990. Fuyant les combats, elles sont également plusieurs dizaines de milliers à avoir été déplacées à l'intérieur du pays par les autorités de Phnom Penh ${ }^{5}$. Toutefois, le niveau actuel des engagements militaires ne peut pas, à lui seul, expliquer l'ampleur de ces mouvements de population. C'est, en effet, la première fois depuis douze ans que l'on assiste à un tel phénomène. D'ailleurs, la guerre actuelle est relativement peu coûteuse en vies humaines ${ }^{6}$, et l'on observe que la première cause d'hospita1isation au Cambodge n'a plus pour origine les blessures par balle mais celles provoquées par les mines. Ces blessures nécessiteraient, malgré tout, selon les données recueillies par les organisations humanitaires à l'intérieur du pays, pas moins de 800 à 1500 amputations par mois. Au risque d'attiser le ressentiment antigouvernemental dans les campagnes, ces mesures plus ou moins autoritaires de regroupement de population sont aussi un signe de faiblesse du gouvernement de M. Hun Sen. Sur le plan tactique, elles ne sont pas sans rappeler la politique des "hameaux stratégiques" autrefois pratiquée en Indochine, dont chacun se souvient de la bien piètre efficacité. Ne pouvant ni déloger ni contenir politiquement les Khmers rouges, le gouvernement central veut leur ôter toute possibilité de recrutement et d'approvisionnement en vivres. Dans la perspective d'élections générales, c'est aussi le moyen le plus sûr de contrôler les populations.

3 Les Khmers rouges sont demeurés malgré leur passé sanglant la seule force de l'opposition au gouvernement pro-vietnamien de Phnom Penh, capable non seulement de le déstabiliser mais peut-être aussi de le renverser. Certes, cette sinistre éventualité est peu probable à court terme. Toutefois, si la situation actuelle continuait à se dégrader, ce qui est inimaginable aujourd'hui pourrait bien devenir réalité dans cinq ou six ans. Malheureusement, ce que déclarait, en mai 1987, le prince Sihanouk dans une interview à un organe de presse américain est encore d'actualité : "Sans les Khmers rouges, la résistance n'a pas de crédibilité sur le champ de bataille et ils sont la seule farce crédible ${ }^{7 "}$. "Pis si l'on croit des estimations faites par la CIA au cours de l'été 1990 dans la perspective d'élections nationales, les Khmers rouges recueilleraient plus du tiers des votes. Espérons au moins que la communauté internationale n'aura pas concouru à chasser les Vietnamiens du Cambodge pour y voir les Khmers rouges reprendre le pouvoir alors qu'ils sont responsables de la mort de plusieurs centaines de milliers de personnes, à l'époque où ils géraient les affaires !

4 Cette armée khmère rouge est organisée en quatre fronts (fonctionnant, virtuellement, de manière autonome et ayant chacun un commandement propre) et se composerait d'un.e vingtaine de brigades, soit 15000 combattants, soutenus par 10000 auxiliaires et 20000 porteurs recrutés chez les 60000 à 100.000 réfugiés sous leur contrôle. Une force considérable, disciplinée et mieux armée que les deux autres composantes noncommunistes de la résistance. Bien que les soldats nationalistes de M. Son Sen aient réussi, cette année, quelques opérations inattendues, leurs dissensions internes réduisent, pour une bonne part, le crédit politique de ces actions. Mais, paradoxalement, c'est au moment où la résistance non communiste obtient quelques succès que la Chine lui coupe ses approvisionnements. Une manière explicite d'empêcher les forces non communistes d'apparaître crédible sur le plan militaire et d'imposer les Khmers rouges comme allié politique et militaire sûr. Beijing n'a en rien 
favorisé la stratégie nationaliste visant à établir ses propres zones libérées. Mais les problèmes de discipline militaire sont tels, dans les "zones libérées" par le FNLPK notamment, que les populations sous son contrôle ont cru bon de faire appel, à plusieurs reprises, aux soldats khmers rouges pour rétablir l'ordre et mettre un terme aux exactions des soldats de la guérilla (vols, viols, etc.).

Bien que les forces non communistes nient l'existence de tout accord de coordination avec les Khmers rouges, le prince Ranariddh affirme, lui, qu'il a eu "un échange de vues, dès le 10 octobre 1989, avec le chef militaire des hommes de Pol Pot - Son Sen pour mener à bien les opérations militaires de la fin de l'année 1989. Le 16 juin 1990, la résistance annonçait même la prise de Kompong Thom après une offensive combinée des trois factions. La coopération militaire entre les factions non communistes et les Khmers rouges semble plus fréquente dans le nord-ouest du pays que sur le front SudOuest dirigé par Pol Pot. Il est certain, par ailleurs, que les Khmers rouges multiplieront d'autant plus leurs actions propres qu'ils auront besoin d'accroître "leur capacité de négociation".

A la conquête de l'Ouest

6 En voulant affronter les forces de la résistance dans la région de Siem Reap, le gouvernement de Phnom Penh veut surtout prévenir toute tentative d'assaut contre la région d'Angkor Vat. Pour se préparer au combat, les Khmers rouges viennent de tracer, au bulldozer, des centaines de kilomètres de pistes dans la forêt. Certes, l'ensemble de ce périmètre (400 monuments sur $300 \mathrm{~km} 2$ ) a été défini comme zone de "non-hostilité" dans le communiqué commun Hun Sen - Sihanouk à Tokyo, mais les Khmers rouges, qui ont juré de conquérir ce symbole national de la puissance khmère passée, ne s'étaient pas associés à cette décision. Cette région est si importante que l'on y a vu, voici quelques semaines, des experts militaires soviétiques, dont on sait qu'ils continuent à entraîner les forces frontalières et les commandos de police de Phnom Penh ${ }^{8}$, dans cette zone. Comme le reconnaît le général Sopheap : "Nous opérons le jour, mais nous sommes obligés de nous retirer la nuit ${ }^{9 "}$. De l'autre côté, les responsables de la résistance ne cachent pas qu'ils aimeraient mettre sur pied une stratégie "de tenaille" selon laquelle les forces nationalistes avanceraient suivant un axe NordOuest/ Sud-Est alors que les Khmers rouges poursuivraient leurs attaques sur le front SudOuest/Nord-Est. Pour ce faire, les combattants khmers rouges s'appuient sur deux axes de pénétration à partir de leurs bases thaïlandaises autour du camp d'O Trao et de la région de Trat (Site 8, Boraï et Site K). Seulement, pour obtenir de meilleurs résultats, dans les mois à venir, il est indispensable que la résistance améliore ses réseaux logistiques pour amener le matériel et les hommes le plus loin possible à l'intérieur du pays. Bien évidemment, une telle évolution signifierait que la résistance aura réussi à se passer de ses bases arrières en Thaïlande, une stratégie de toute première importance au moment où Bangkok envisage de fermer sa frontière avec le Cambodge. Il ne faut pas oublier que, depuis 1988, une tension assez vive s'est manifestée entre Thaïlandais et Khmers rouges lorsque ces derniers ont tenté de rapatrier leurs stocks d'armes à l'intérieur du Cambodge. C'est pourquoi ils chercheraient également à disposer d'un accès direct à la mer au cas où Bangkok cesserait de laisser transiter les armes sur son sol. Ces coups de mains accréditent également le fait qu'ils peuvent attaquer là où ils veulent quand ils veulent ; autrement dit, nul ne peut continuer à ignorer leur réalité dans les négociations internationales et à les évincer totalement d'une solution politique du conflit. 
Les aspects économiques de la stratégie khmère rouge

7 A l'heure actuelle, on peut dire qu'il existe deux guerres au Cambodge, l'une politique et militaire, et l'autre essentiellement économique. En massant près de 8000 de leurs hommes dans la région de Paillin, les Khmers rouges montrent combien cette partie du pays est importante pour eux. Si les enjeux militaires de cette région sont, somme toute, mineurs pour les belligérants, son importance économique est considérable pour les Khmers rouges. En effet, cette province renferme des gisements de "rubis du Siam" et de grenats qui leur permettent de tirer des revenus substantiels pour leurs opérations d'achats d'armes et de nourriture. Un homme d'affaires thaïlandais affirmait récemment, à Bangkok, avoir remis 24 millions de bahts aux Khmers rouges, au cours des derniers mois, pour des concessions minières ${ }^{10}$. En effet, les trafiquants thaillandais ou birmans, qui se précipitent dans la région, doivent payer 500 à 1000 bahts par mineur pour que ceux-ci puissent s'introduire pendant une semaine dans la zone minière. Il y a, chaque jour, 200 à 300 vendeurs qui viennent s'approvisionner en rubis, ce qui rapporte plusieurs millions de dollars aux Khmers rouges et devrait leur permettre de se passer partiellement de l'aide chinoise. Ces trafiquants craignent, à l'évidence, les tirs d'artillerie et les mines éparpillées dans la région, mais les Khmers rouges ne sont pas pour eux un danger. Disciplinés, ceux-ci ne les volent pas. Hormis le trafic de pierres précieuses, de très hauts cadres Khmers rouges se livrent encore à d'autres pratiques douteuses comme le commerce illégal de bois précieux. Des entreprises thailandaises qui ont signé des actes de concession avec Ieng Sary auraient même demandé au ministère thaillandais des finances des autorisations d'exploitation et d'exportation. Les trafics sont si nombreux sur la frontière, et les complicités importantes au sein de l'armée thaillandaise, qu'il s'agit maintenant d'intéresser au sens économique du terme les militaires de Bangkok à une solution politique du conflit khmer.

8 Les intérêts économiques parfois divergents entre les brigades khmères rouges ont fait naître occasionnellement des conflits qui se sont réglés par les armes. Dès lors, pourquoi être surpris du petit nombre d'attaques sur la route N4 qui relie Kompong Som à la capitale, sur laquelle transitent les 2/3 des approvisionnements militaires du régime pro-vietnamien et la quasi-totalité de ses livraisons énergétiques? Les quelques commerçants faisant la navette entre les deux villes vous avoueront, d'ailleurs, qu'ils paient régulièrement les Khmers rouges pour qu'ils laissent la route ouverte. Dans leur stratégie d'autonomie économique, les Khmers rouges font une place de plus en plus importante au trafic de drogue, ce qui n'est pas sans inquiéter les autorités thaillandaises. Durant l'été, $100 \mathrm{~kg}$ de marijuana cultivée par les Khmers rouges dans des champs en terre thailandaise ont, par exemple, été saisies ${ }^{11}$. Ainsi, pour affaiblir l'économie nationale et ruiner les efforts de libéralisation de ces dix-huit derniers mois, les Khmers rouges viennent-ils de mettre en garde les gouvernements et les sociétés étrangères signant des accords de coopération avec les autorités de Phnom Penh. Le prince Sihanouk avait, lui aussi, proféré il y a quelques années de telles menaces. Mais, aujourd'hui, la concomitance de cette annonce ${ }^{12}$ et la visite à Phnom Penh de M. Thierry de Beaucé - secrétaire d'État aux relations culturelles internationales - ainsi que la décision de Paris d'autoriser Air Liberté à ouvrir une liaison aérienne régulière avec Phnom Penh, sonne comme une mise en garde contre la France. Le deuxième volet économique de la guerre, consiste à obliger les autorités de Phnom Penh à consacrer toujours plus de moyens à l'effort de guerre pour ralentir la reconstruction économique qui offrirait un surcroît de légitimité au régime. En l'absence du soutien 
économique et financier des pays de l'ex-bloc socialiste, l'administration de Phnom Penh dispose de moins en moins de moyens pour continuer à exister Ces difficultés sont d'autant plus importantes que le régime pro-vietnamien doit consacrer près de $40 \%$ de son budget annuel à sa défense, au détriment de secteurs vitaux comme l'agriculture. En réduisant à néant les efforts visant à mettre sur pied les infrastructures de transport, notamment, c'est l'ensemble des efforts économiques qui sont remis en cause.

Une victoire militaire khmère rouge est-elle envisageable ? Plutôt que de se lancer dans des offensives de grandes envergures comme leurs alliés du FNLPK, les Khmers rouges préfèrent opérer selon les règles classiques de la guérilla. En effet, une guerre conventionnelle est très coûteuse en hommes. Le FNLPK en a fait la dure expérience au cours de l'hiver 1989-1990 en s'évertuant à attaquer des localités très bien défendues par des opérations où il a perdu beaucoup d'hommes. La stratégie khmère rouge a, elle, pour but essentiel d'asphyxier Phnom Penh en coupant les réseaux de communication : attaques sur la route du port maritime de Kompong Som, la région de Koh Kong et le chemin de fer qui relie la capitale à Battambang ${ }^{13}$. Phénomène nouveau, les Khmers rouges se déplacent en unités plus importantes. Ils sont passés, au cours de la dernière année, de petites unités d'une dizaine d'individus à d'autres de plusieurs centaines aujourd'hui. Jamais, depuis 1978, leurs actions ne sont venues jauger de si près les défenses de la capitale cambodgienne (à moins de $50 \mathrm{~km}$ ), et jamais ils ne se sont approchés si nombreux de la frontière vietnamienne (des accrochages ont été signalés en septembre dans le nord de la province de Svay Rieng). Pour contrer cette politique d'infiltration, l'armée de Phnom Penh a donc décidé de faire descendre des montagnes ses unités qui y étaient stationnées pour que celles-ci s'installent dans les rizières. En définitive, l'offensive khmère rouge n'a pas pour but de s'emparer de territoires mais de désorganiser l'appareil administratif et économique national. Cette stratégie nécessite, pour le moment, de ne jamais accepter un engagement frontal où l'infériorité numérique risquerait de lui être fatale.

Outre l'insuffisance de son commandement, l'armée de Phnom Penh se contente, elle, de défendre les villes en s'appuyant sur sa supériorité en puissance de feu mais n'a jamais cherché à profiter de celle-ci ou de sa supériorité numérique. Les conseillers militaires soviétiques se sont d'ailleurs plaints à plusieurs reprises de cette passivité des soldats et du commandement khmers. Selon les militaires thaillandais sur la frontière, les Khmers rouges seraient capables de s'emparer de la ville de Siem Reap mais ne disposeraient pas des forces pour la conserver. De plus, les protecteurs chinois ne souhaitent pas apparaître comme des va-t-en-guerre au moment où leur diplomatie cherche à sortir la Chine de son isolement international. En août 1990, dans le cadre d'un plan de règlement global, les Cinq - dont la Chine - avaient d'ailleurs appelé les Cambodgiens à faire preuve de retenue avant la proclamation formelle d'un cessez-lefeu.

11 Sur le terrain, les soldats gouvernementaux recrutés de force, mal payés et peu disciplinés, ne disposent pas d'un soutien populaire considérable même s'ils font face aux Khmers rouges, de sinistre mémoire. Quant à ceux-ci, le peu que l'on connaisse de leur recrutement ne permet pas de laisser croire que leurs pratiques soient uniquement coercitives. Il semble même que, sur chaque front khmer rouge, les pratiques de recrutement soient différenciées. Dans la zone Sud-Ouest, Pol Pot ne recruterait que des combattants volontaires auxquels une formation idéologique de "qualité" serait 
imposée. Dans ce cas, il ne faut pas s'étonner que les combattants d'élite (cf. les forces spéciales) et les plus déterminés soient réunis dans cette zone. Quant aux hommes de Ta Mok, alias Chhit Choeun, plus au nord, priorité est donnée à la formation de guerriers plutôt qu'à des instructeurs politiques. De manière générale, les hommes de Pol Pot sont plus politisés, plus motivés que leurs camarades d'autres zones khmères rouges. Cela ne veut pas dire que, dans leurs rangs, il n'y ait pas de déserteurs. Dans un certain nombre d'unités, on a pu remarquer que le moral des troupes était très faible ; les combattants arguent que leurs dirigeants se battent entre eux et qu'ils ne sont plus certains de pouvoir gagner la guerre. L'absence de nourriture et la crainte des mines sont aussi les causes de ces départs, dont il est bien difficile d'évaluer l'importance. Même les prisonniers de guerre capturés sont plus souvent rééduqués que passés par les armes et peuvent être ainsi réutilisés comme des supplétifs. En l'absence de visite du comité international de la Croix-Rouge aux prisonniers, il est bien difficile de savoir comment ils sont traités, tout comme on ignore le sort réservé aux déserteurs.

en déplaise, les Khmers rouges disposent dans tout le pays d'une véritable aura ou, au moins, de la bienveillante neutralité d'un très grand nombre. Les Khmers rouges qui paient, eux, toutes les denrées dont ils ont besoin ne sont pas nécessairement en secteur hostile. Ouvertement, certains d'entre eux participent aux travaux des champs.

Dans les régions où ils sont actifs, les Khmers rouges ne liquident pas systématiquement l'administration en place et font même peu d'efforts pour construire immédiatement une nouvelle structure administrative. Dans les villages "libérés", les séances de formation politique sont moins nombreuses qu'autrefois. Dans leurs discours, ils disent se battre pour libérer le pays des Vietnamiens, ce qui est loin d'être impopulaire, tout comme l'est l'utilisation permanente de l'image du prince Sihanouk, le "dieu-roi" du Cambodge. La propagande est toute simple: ils sont les seuls vrais patriotes de la nation, les autres sont des "vendus" aux Vietnamiens. On déclare aboli le régime de Pol Pot et on prétend que le choix des leaders se fera par vote populaire.

Quant aux liquidations de fonctionnaires, elles ne provoquent pas le tollé que l'on croit. Choisissant leurs victimes parmi les responsables les plus corrompus du régime, les Khmers rouges ne font pas toujours peur Pour autant, cela ne veut pas dire qu'ils aient changé ${ }^{14}$. Mais, selon les rares informations dont on dispose, $80 \%$ des combattants de 1990 n'étaient pas dans les rangs khmers rouges quand ceux-ci étaient au pouvoir. Dirigés par les vétérans du Kampuchéa démocratique, les combattants khmers rouges sont probablement aujourd'hui plus jeunes, moins motivés et moins expérimentés que ceux auxquels durent faire face les Vietnamiens au début de la guerre. Quant à leur idéologie maoïste, avant-gardiste, ceux qui les ont approchés, ces dernières années, conviennent tous que, sur le fond de leur programme, rien n'a changé en dépit des déclarations tonitruantes de certains leaders clamant leur conversion aux bienfaits de la démocratie parlementaire et de l'économie libérale. Contrairement aux affirmations de Khieu Samphan ${ }^{15}$, Pol Pot reste le chef incontesté des Khmers rouges et dirige avec Nuon Chea, le numéro trois ${ }^{16}$, le bureau 87 , c'est-à-dire le commandement suprême des Khmers rouges.

Pour infiltrer plus facilement une région, la plus grande qualité des Khmers rouges, sans conteste jusqu'à présent, est leur patience et leur connaissance du terrain. Avant de monter un coup de main, les Khmers rouges étudient pendant plusieurs jours, voire plusieurs semaines, le rapport des forces. Ils n'attaquent que le jour où ils sont sûrs de remporter la victoire. Autrement dit, l'organisation du renseignement militaire est de 
toute première importance. C'est pourquoi, depuis 1985-1986, les responsables khmers rouges s'appuient en général sur des combattants locaux pour développer leurs actions. Dans la région de Kampot, ce sont donc des hommes originaires de la province qui harcèlent les forces régulières et organisent le travail politique dans les villages. C'est pour répondre à ces nouveaux dangers que Phnom Penh réorganisa, elle aussi, dans le courant 1989, son dispositif militaire. Dans la perspective d'affrontements croissants entre les troupes de Phnom Penh et la guérilla khmère rouge, Moscou a livré beaucoup d'armes légères et de munitions devant équiper, avant tout, les milices locales que Hun Sen souhaitait vouloir plus utilisées dans ce qu'il appelle la "guerre populaire". Ainsi, les milices se voient confier, de plus en plus, la défense au niveau des villages alors que l'armée régulière a été regroupée autour des commandements provinciaux. Le commandement général espère que ses hommes se battrant mieux, car ils devront "défendre leur famille et leur village" et seront mieux à même de contrer les opérations d'infiltration khmères rouges, une motivation supplémentaire qui devait aussi suppléer la modicité des soldes versées. Pour autant, les désertions sont toujours aussi nombreuses. Une des causes majeures de ces désertions, du côté de Phnom Penh, est le recrutement obligatoire de "Volontaires".

La loi, édictée le 3 août 1989, qui prévoit une conscription triennale, obligatoire pour tous les hommes de 18 à 35 ans, fait perdre, sans conteste, beaucoup de crédit au gouvernement Hun Sen $17^{17}$. De plus, en l'absence de véritable état civil au Cambodge, le seul moyen de faire appliquer la loi est de procéder à de véritable rafles, ce qui, évidemment, est loin d'être populaire et n'est pas sans rappeler ce qui se passait à la fin du régime Lon Nol. Dans le même temps, l'État du Cambodge n'a pas réussi à se rallier les combattants de la résistance. A croire que les mesures prises lors du 10ème anniversaire de la RPK visant à doubler les récompenses prévues pour les personnes "égarées qui retourneraient à la révolution" en apportant des armes, du matériel de guerre et/ou des documents importants ne suffisaient pas !

Une solution rouge pour le Cambodge?

Le régime en place à Phnom Penh est tellement corrompu et impopulaire que beaucoup d'observateurs n'hésitent pas à comparer celui-ci à son prédécesseur républicain (Lon Nol : 1970-1975). Incapable d'assurer seul sa sécurité face aux 40000 combattants de la résistance, le régime Hun Sen - Heng Samrin a dû, de nouveau, faire appel à 25000 bodoïs pour assurer sa survie. Hanoi, qui doit également faire face à une crise économique profonde née des erreurs passées, demande à Phnom Penh de 100 à 150 dollars par soldat vietnamien et par mois de présence effective au Cambodge. Un argument non négligeable pour la résistance qui dénonce et base sa propagande politique sur cette intervention étrangère. S'il paraît évident que jamais les conseillers militaires vietnamiens n'ont quitté leurs postes auprès du Premier ministre, des ministres de la Défense et de l'Intérieur, la présence de plusieurs dizaines de civils vietnamiens dans le pays nourrit un profond ressentiment contre le gouvernement. Le plus inquiétant pour beaucoup, aujourd'hui, serait une "solution rouge". De récents contacts directs entre Beijing et Phnom Penh, à Bangkok, ont relancé les spéculations sur une telle éventualité basée sur un accord politique entre le parti communiste au pouvoir dont certains des leaders sont d'anciens khmers rouges (Heng Samrin, Hun Sen, Chea Sim) et les Khmers rouges. Selon diverses sources à Hanoi, Beijing et Bangkok, des éléments du PPRK, qui serait constitué dans une forte proportion (impossible toutefois à évaluer) d'anciens khmers rouges, explorent cette possibilité pour préserver le caractère communiste du Cambodge tout en mettant un terme au conflit armé. Une telle 
hypothèse n'est pas complètement farfelue ni nouvelle, les Soviétiques auraient même proposé à la Chine, en décembre 1987, d'organiser une rencontre Hun Sen - Khieu Samphan ${ }^{18}$. D'ailleurs, le nouveau ministre des Affaires étrangères, M. Hor Nam Hong (un proche de Hun Senj, a admis que "cette idée a germé dans l'esprit de certains"19, sans compter que les Vietnamiens ont accepté, à maintes reprises, de prendre langue avec les Khmers rouges, à l'exception des leaders. De telles spéculations sont, aujourd'hui, renforcées par la fragilité du Premier ministre et les arrestations visant à mettre un terme à toute tentative de multipartisme. M. Hun Sen, dont on dit qu'il serait en minorité au bureau politique, doit faire face à une campagne de reprise en main de l'appareil du PPRK et au durcissement, au plan et devra même s'expliquer intérieur (ex. lutte contre la corruption), sur sa politique, pour la première fois, devant le Parlement. Dans un tel contexte, qui peut encore croire que la paix est proche, que les rapatriements des populations réfugiées sont imminents et que, demain, la tenue d'élections constitue les prémices du retour à la démocratie? Finalement, il est à craindre que les offensives de cette saison sèche ne changent rien, fondamentalement, à la situation générale sur le terrain et que les Grands, quoique "préoccupés" par l'absence de progrès politique dans les discussions intercambodgiennes, soient dans l'incapacité totale d'imposer la paix.

\section{NOTES}

1. The Mail on Sunday 6 octobre 1990 - AFP 6 octobre 1990 - The Independent, 27 octobre 1990.

2. Les commandos d'élite de l'armée britannique.

3. Bangkok Post, 17 septembre 1990.

4. Front national de libération du peuple Khmer.

5. En septembre 1990 on avançait le chiffre de 150000 personnes déplacées de 9 provinces sur les 20 que compte le pays. Seulement 20000 personnes parmi ces réfugiés auraient pu regagner leur village.

6. Les Khmers rouges auraient tué entre 7000 et 8 000personnes de 1979 à 1989.

7. Cité par Frank Tatu in Cambodia : A Country Study, US Government printing, Washington, 1990, p. 269.

8. Washington Times, 17 mai 1990.

9. AFP, 25 octobre 1990.

10. The Economist, 25 novembre $1989-1$ baht $=0,24 \mathrm{FF}$

11. 11. Bangkok Post, 13 juillet 1990 - Agence Cambodge-Laos, Paris, $n^{\circ}$ 18, octobre 1990.

12. AFP, 23 octobre 1990.

13. Bangkoh Post 21 octobre 1990 : depuis 1979 les autorités de Phnom Penh ont relevé 72 attaques de train du fait des Khmers rouges. En 1990, celles-ci ont tué 50 personnes dans la région de Kampot (20 octobre), 26 à Kompong Trach (1er juillet) et 30 autres à Kompong Chhnang (15 juillet).

14. Roger Normand, The Teachings of Chairman Pot, The Nation, New York, 27 août, 3 septembre 1990, p. 198-202. 
15. Stern, 19 septembre 1989.

16. Le $n^{\circ} 2$ est Ieng Sary le $n^{\circ} 4$ est Son Sen.

17. Sont exemptés normalement les chefs de famille, les étudiants (le départ des enseignants soviétiques et la fermeture de nombre d'écoles modifient profondément cet avantage), les orphelins, les membres des familles dont un proche a été tué au combat depuis moins d'un an, les handicapés, les blessés, les criminels, ainsi que "les citoyens dont la conduite ne retient pas la confiance du peuple". [[Agence Cambodge Laos, Paris, $\mathrm{n}^{\circ} 15$, novembre 1989.

18. Far Eastern Economic Review, 8 juin 1988

19. AFP, 27 octobre 1990.

\section{RÉSUMÉS}

Les cambodgiens s'affrontent entre eux sans que cela soit un enjeu stratégique planétaire ni même régional. Les combats n'en continuent pas moins et les Khmers rouges sont en passe de triompher sur le terrain avec l'appui en armement des chinois. Ils contrôlent comme les autres groupes de résistance les populations réfugiées dans les camps et les déplacent en fonction de leur stratégie. Ils jouent aussi des ressources tirées de l'aide humanitaire et ne peuvent être évincés d'une solution politique car sur le terrain, ils sont de loin la composante principale de l'opposition au pouvoir pro-Vietnamien. Au delà de la stratégie militaire, les Khmers rouges ont une stratégie économique qui passe par des trafics de pierres précieuses... et qui leur permet d'obtenir des revenus substanciels pour acheter des armes et de la nourriture. Ils dépendent ainsi de moins en moins de l'extérieur et peuvent poursuivre la guerre durant très longtemps s'ils le désirent.

The continuing Cambodian civil war has no world or regional strategic consequences. The fights are nonetheless still going on, and the Khmers are about to triumph thanks to the weapons furnished by China. Like other resisting groups, they control refugees living in camps and displace them according to their strategy. They play with humanitarian aid and cannot be ignored in case of a political solution since they are the main opposition group to the proVietnamese government. Apart from their military strategy, the red Khmers also have an economic one which enables them to buy arms and food through a gems traffic. Thus, they depend less and less on the international community, and can make the war last for as long as they want.

\section{INDEX}

Mots-clés : conflits, génocide, guerre

Index géographique : Asie du Sud-Est, Cambodge

Index chronologique : 1990 - 2000

Thèmes : Khmers rouges 
AUTEUR

FRANÇOIS GUILBERT

Economiste, chercheur à l'Institut de Relations Internationales et Stratégiques, enseignant à

l'Université Paris I. 